\title{
Slave-Boson Mean-Field Theory of the Antiferromagnetic State in the Doubly Degenerate Hubbard Model - The Half-Filled Case -
}

\author{
Hideo Hasegawa ${ }^{\dagger}$ \\ Department of Physics, Tokyo Gakugei University \\ Koganei, Tokyo 184, Japan
}

(February 5, 1997)

\begin{abstract}
The antiferromagnetic ground state of the half-filled Hubbard model with the doubly degenerate orbital has been studied by using the slave-boson mean-field theory which was previously proposed by the present author. Numerical calculations for the simple cubic model have shown that the metal-insulator transition does not take place except at the vanishing interaction point, in strong contrast with its paramagnetic solution. The energy gap in the density of states of the antiferromagnetic insulator is much reduced by the effect of electron correlation. The exchange interaction $J$ plays an important role in the antiferromagnetism: although for $J=0$ the sublattice magnetic moment $m$ in our theory is fairly smaller than $m_{\mathrm{HFA}}$ obtained in the Hartree-Fock approximation, $m$ for $J / U>0.2\left(U\right.$ : the Coulomb interaction) is increased to become comparable to $m_{\mathrm{HFA}}$. Surprisingly, the antiferromagnetic state is easily destroyed if a small, negative exchange interaction $(J / U<-0.05)$ is introduced.
\end{abstract}

PACS No. 71.27+a, 71.30.+h, 75.10.Lp 


\section{INTRODUCTION}

Much progress has been made in our theoretical understanding on the effect of electron correlation in systems such as transition metals and high- $T_{c}$ materials. Most of the theoretical studies have been made for the single-band Hubbard model $(\mathrm{SHM})^{1-3}$ for its simplicity. Actual systems, however, inevitably have the orbital degeneracy. It is necessary to investigate the role of the orbital degeneracy and the effect of Hund-rule coupling due to the exchange interaction for a better understanding on strongly correlated systems.

In the last few years the Hubbard model with orbital degeneracy has been extensively studied by using various methods such as the Gutzwiller approximation (GA), ${ }^{4-6}$ the slave-boson theory ${ }^{7,8}$, the dynamical mean-field approximation, ${ }^{9}$ and the projective self-consistent method. ${ }^{10}$ The original GA proposed by Gutzwiller and Chao ${ }^{11}$ was reformulated in Refs.4-6. In a previous paper ${ }^{7}$ (referred to as I hereafter), the present author developed the slave-boson functional-integral method for the Hubbard model with an arbitrary, orbital degeneracy, by employing the method proposed by Dorin and Schlottman ${ }^{12}$ for the Anderson lattice model. Frésard and $\operatorname{Kotliar}^{8}$ developed an alternative slave-boson functional integral method. These slave-boson theories are the simple generalization of the Kotliar-Ruckenstein theory for the $\mathrm{SHM}^{13}$ to that for the degenerate Hubbard model, and their saddle-point approximation is equivalent to the GA. ${ }^{4-6,11}$ In I we have studied the metal-insulator (MI) transition of the doubly degenerate Hubbard model (DHM) in the paramagnetic state. The MI transition takes place when the interaction strength is increased, ${ }^{4,6,9,10}$ just as in the case of the SHM. ${ }^{14}$ This MI transition is shown to become the first-order one in the half-filled case when the exchange interaction is included. ${ }^{6,7}$

We should, however, remind the following facts having been established for the half- 
filled SHM:

(a) In the paramagnetic $(\mathrm{P})$ state, the MI transition is realized for $d=1,2,3$ and $\infty$ in the GA, but not for $d=1,2$ and 3 in the advanced theory going beyond the GA. ${ }^{15-17}$

(b) In the antiferromagnetic (AF) state, the MI transition occurs in neither $d=1$, 2, nor $3^{18}$ in the advanced theory, nor in $d=\infty$ even within the GA. ${ }^{19-22}$

These facts suggest that it is indispensable to take into account the antiferromagnetic state in discussing the MI transition in DHM.

One of the advantages of the slave-boson functional integral method over the GA is that it has the wider applicability than the GA. For example, we can deal with the system with the complicated magnetic structures such as the antiferromagnetic state, by using the Green's function formalism. We will study in this paper, the antiferromagnetic state of the DHM by employing our slave-boson mean-field theory, ${ }^{7}$ in order to clarify the above-mentioned issue relevant to the MI transition and the roles of the degeneracy and the exchange interaction.

The paper is organized as follows: In the next Sec.II, we present a basic formulation of our slave-boson saddle-point approximation to deal with the antiferromagnetic state in the DHM, after briefly reviewing I. Numerical calculations for the simple-cubic lattice are presented in Sec.III. Section IV is devoted to conclusion and supplementary discussion.

\section{FORMULATION}

We adopt the Hubbard model with the arbitrary, orbital degeneracy $D$, whose Hamil- 
tonian is given by

$$
H=\sum_{\sigma} \sum_{i j} \sum_{m m^{\prime}} t_{i j}^{m m^{\prime}} c_{i m \sigma}^{\dagger} c_{j m^{\prime} \sigma}+\frac{1}{2} \sum_{i} \sum_{(m, \sigma) \neq\left(m^{\prime}, \sigma^{\prime}\right)} U_{m m^{\prime}}^{\sigma \sigma^{\prime}} c_{i m \sigma}^{\dagger} c_{i m \sigma} c_{i m^{\prime} \sigma^{\prime}}^{\dagger} c_{i m^{\prime} \sigma^{\prime}}
$$

where $c_{i m \sigma}$ is an annihilation operator of an electron with an orbital index $m$ and spin $\sigma(=\uparrow, \downarrow)$ on the lattice site $i$. The electron hopping is assumed to be allowed only between the same sub-band: $t_{i j}^{m m^{\prime}}=t_{i j} \delta_{m m^{\prime}}$, for a simplicity. The on-site interaction, $U_{m m^{\prime}}^{\sigma \sigma^{\prime}}$, is given by

$$
\begin{aligned}
U_{m m^{\prime}}^{\sigma \sigma^{\prime}} & =U_{0}=U \quad \text { for } m=m^{\prime}, \sigma \neq \sigma^{\prime}, \\
& =U_{1}=U-2 J \text { for } m \neq m^{\prime}, \sigma \neq \sigma^{\prime}, \\
& =U_{2}=U-3 J \text { for } m \neq m^{\prime}, \sigma=\sigma^{\prime},
\end{aligned}
$$

where $U$ and $J$ are Coulomb and exchange interactions, respectively.

In I we employed the boson opertor intoduced by Dorin and Schlottman, ${ }^{12}$ and used the static approximation to get the functional integral representation of the partition function given $b^{7}$

$$
Z=\int D \xi \int D \nu \int D m \int D n \int \Pi_{\ell=2}^{2 D} D b^{(\ell)} \exp (-\beta \Phi)
$$

with

$$
\begin{aligned}
e^{-\beta \Phi} & =\exp \left(-\beta\left[\sum_{i} \sum_{m}\left(\xi_{i m} m_{i m}-\nu_{i m} n_{i m}\right)+\Phi_{0}\right]\right) \operatorname{Tr} \exp \left(-\beta H_{e f f}\right), \\
\Phi_{0} & =\sum_{i} \sum_{\ell=2}^{2 D} \sum_{m_{1} \sigma_{1}, \ldots, m_{\ell} \sigma_{\ell}} \sum_{\left(m \sigma, m^{\prime} \sigma^{\prime}\right)} U_{m m^{\prime}}^{\sigma \sigma^{\prime}} b_{i ; m_{1} \sigma_{1}, \ldots ., m_{\ell} \sigma_{\ell}}^{(\ell) \dagger} b_{i ; m_{1} \sigma_{1}, \ldots ., m_{\ell} \sigma_{\ell} .}^{(\ell)}
\end{aligned}
$$

The effective Hamiltonian is given by

$$
H_{e f f}=\sum_{\sigma} \sum_{i j} \sum_{m} z_{i m \sigma}^{\dagger} z_{j m \sigma} t_{i j} c_{i m \sigma}^{\dagger} c_{j m^{\prime} \sigma}+\sum_{\sigma} \sum_{i} \sum_{m}\left(\nu_{i m}-\sigma \xi_{i m}\right) c_{i m \sigma}^{\dagger} c_{i m \sigma}
$$


with

$$
z_{i m \sigma}=2\left(2-n_{i m}\right)^{-1 / 2} n_{i m}^{-1 / 2}\left[\sqrt{\bar{e}_{i} p_{i m \sigma}}+\sqrt{\overline{p_{i m \sigma}}} b_{i}^{(2)}+\sum_{\ell=3}^{2 D}\left(b_{i}^{(\ell-1)} \cdot b_{i}^{(\ell)}\right)_{m \sigma}\right]
$$

In Eqs.(5)-(9), $D \xi=\prod_{i m} \xi_{i m}$ et al., $\xi_{i m}\left(\nu_{i m}\right)$ is the exchange (charge) field in the subband $m$ at the site $i$, and $m_{i m}\left(n_{i m}\right)$ is the magnetic moment (electron number). The forth summation in eq. (7) is performed over a pair of indices $\left(m \sigma, m^{\prime} \sigma^{\prime}\right)$ with $(m \sigma) \neq\left(m^{\prime} \sigma^{\prime}\right)$ in the configuration: $\left\{m_{1} \sigma_{1}, m_{2} \sigma_{2}, \ldots, m_{\ell} \sigma_{\ell}\right\}$ occipied by $\ell$ electrons. The introduced boson operator, $b_{i}^{(\ell)}$ :

$$
b_{i}^{(\ell)} \equiv b_{i ; m_{1} \sigma_{1}, \ldots, m_{\ell} \sigma_{\ell}}^{(\ell)}
$$

projects to the configuration of $\ell$ electrons with pairs of orbital and spin indices $\{m \sigma\}$. Its full contraction, $\left(b_{i}^{(\ell)} \cdot b_{i}^{(\ell)}\right)$, and partial contraction, $\left(b_{i}^{(\ell)} \cdot b_{i}^{(\ell)}\right)_{m \sigma}$, are defined by

$$
\begin{gathered}
\left(b_{i}^{(\ell)} \cdot b_{i}^{(\ell)}\right) \equiv \sum_{m_{1} \sigma_{1}, \ldots ., m_{\ell} \sigma_{\ell}} b_{i ; m_{1} \sigma_{1}, \ldots ., m_{\ell} \sigma_{\ell}}^{(\ell)} b_{i ; m_{1} \sigma_{1}, \ldots, m_{\ell} \sigma_{\ell}}^{(\ell)}, \\
\left(b_{i}^{(\ell)} \cdot b_{i}^{(\ell)}\right)_{m_{n} \sigma_{n}} \equiv \sum_{m_{1} \sigma_{1}, m_{2} \sigma_{2}, \ldots ., m_{n-1} \sigma_{n-1}, m_{n+1} \sigma_{m+1} 1, \ldots ., m_{\ell} \sigma_{\ell}} b_{i ; m_{1} \sigma_{1}, m_{2} \sigma_{2}, \ldots, m_{\ell} \sigma_{\ell}}^{(\ell)} b_{i ; m_{1} \sigma_{1}, m_{2} \sigma_{2}, \ldots ., m_{\ell} \sigma_{\ell} \cdot}^{(\ell)} .
\end{gathered}
$$

The empty state $\left(e_{i}\right)$ and singly occupied one with a $\sigma$-spin electron $\left(p_{i m \sigma}\right)$ are expressed in terms of $n_{i m}, m_{i m}$ and $b_{i}^{(\ell)}$ for $\ell \geq 2$ as $^{7}$

$$
\begin{gathered}
e_{i}=\left(b_{i}^{(0)} \cdot b_{i}^{(0)}\right)=1-\sum_{m} n_{i m}+\sum_{m \sigma} \sum_{\ell=2}^{2 D}[(\ell-1) / \ell]\left(b_{i}^{(\ell)} \cdot b_{i}^{(\ell)}\right)_{m \sigma} \\
p_{i m \sigma}=\left(b_{i}^{(1)} \cdot b_{i}^{(1)}\right)_{m \sigma}=\left(n_{i m}+\sigma m_{i m}\right) / 2-\sum_{\ell=2}^{2 D}\left(b_{i}^{(\ell)} \cdot b_{i}^{(\ell)}\right)_{m \sigma} .
\end{gathered}
$$

The expression for the functional integral given by eqs.(5)-(14) is a generalization of the single-band model to the degenerated-band model, ${ }^{21)}$ and it has a transparent physical meaning. 
When we apply our slave-boson functional integral method developed in I to the DHM $(D=2)$, the functional integral becomes

$Z=\int D \xi \int D m \int D \nu \int D n \int D d_{0} \int D d_{1} \int \Pi_{\sigma} D d_{\sigma} \int \Pi_{\sigma} D t_{\sigma} \int D f \exp \left[-\beta\left(\Phi_{0}+\Phi_{1}+\Phi_{2}\right)\right]$

$$
\Phi_{0}=\sum_{i}\left[2 U_{0} d_{i 0}+2 U_{1} d_{i 1}+U_{2}\left(d_{i \uparrow}+d_{i \downarrow}\right)+2\left(U_{0}+U_{1}+U_{2}\right)\left(t_{i \uparrow}+t_{i \downarrow}+f_{i}\right)\right]
$$

with

$$
\begin{gathered}
\Phi_{1}=\sum_{i m}\left[\xi_{i m} m_{i m}+\left(\mu-\nu_{i m}\right) n_{i m}\right], \\
\Phi_{2}=\int d \varepsilon f(\varepsilon)(-1 / \pi) \operatorname{Im} \operatorname{Tr} \ln G(\varepsilon) .
\end{gathered}
$$

In Eqs.(15)-(18), $f(\varepsilon)$ is the Fermi-distribution function, and $d_{i}, t_{i}$ and $f_{i}$ denote the states with double, triple and quadruple occupations, respectively. In particular for doubly occupied states, we take into account the three kinds of configurations: $d_{i 0}$ for a pair of electrons on the same orbital with opposite spin, $d_{i 1}$ on the different orbital with opposite spin, and $d_{i \sigma}$ on the different orbital with same spin $\sigma$.

The one-particle Green function, $G(\varepsilon)$, in Eq.(18) is expressed by

$$
G(\varepsilon)=\left(\varepsilon-H_{e f f}\right)^{-1}
$$

where the effective Hamiltonian, $H_{e f f}$, is given by

$$
H_{e f f}=\sum_{\sigma} \sum_{i j} \sum_{m} q_{m \sigma}^{i j} t_{i j} c_{i m \sigma}^{\dagger} c_{j m \sigma}+\sum_{\sigma} \sum_{i} \sum_{m}\left(\nu_{i m}-\sigma \xi_{i m}\right) c_{i m \sigma}^{\dagger} c_{i m \sigma}
$$

the band-narrowing factor, $q_{m \sigma}^{i j}$, being given by

$$
q_{m \sigma}^{i j}=z_{i m \sigma} z_{j m \sigma}
$$


with

$$
\begin{gathered}
z_{i m \sigma}=\frac{2\left[\sqrt{p_{i \sigma}}\left(\sqrt{e_{i}}+\sqrt{d_{i \sigma}}\right)+\left(\sqrt{d_{i 0}}+\sqrt{d_{i 1}}\right)\left(\sqrt{p_{i-\sigma}}+\sqrt{t_{i \sigma}}\right)+\sqrt{t_{i-\sigma}}\left(\sqrt{d_{i-\sigma}}+\sqrt{f_{i}}\right)\right]}{\left(n_{i m}+\sigma m_{i m}\right)^{1 / 2}\left(2-n_{i m}-\sigma m_{i m}\right)^{1 / 2}} . \\
e_{i}=1-2 n_{i m}+2 d_{i 0}+2 d_{i 1}+d_{i \uparrow}+d_{i \downarrow}+4\left(t_{i \uparrow}+t_{i \downarrow}\right)+3 f_{i} \\
p_{i m \sigma}=\left(n_{i m}+\sigma m_{i m}\right) / 2-\left(d_{i 0}+d_{i 1}+d_{i \sigma}\right)-2 t_{i \sigma}-t_{i-\sigma}-f_{i} .
\end{gathered}
$$

In order to discuss the antiferromagnetic (AF) state, we divide the crystal into two sublattices, A and B. We assume that for the $\mathrm{AF}$ wave vector $Q$, the relation: $\varepsilon_{k+Q}=-\varepsilon_{k}$ holds where $\varepsilon_{k}$ is the Fourier transform of the transfer integral, $t_{i j}$. We take $\xi_{i m}$ in Eq.(20) as the staggered field given by $\xi_{i m}=\xi(-\xi)$ for $i \in A(i \in B)$, the exchange fields in the two subbands being assumed to be the same. The magnitude of $\xi$ will be determined by the variational condition, as will be shown shortly (Eq.(31)).

Since the effective transfer integral in Eq.(20) is expressed as a product form: $z_{i m \sigma} t_{i j} z_{j m \sigma}$, we can express the one-electron Green function in terms of the locators defined $\operatorname{by}^{23)}$

$$
X_{i m \sigma}=\left(\varepsilon-\nu_{i m}+\sigma \xi_{i m}\right) / r_{i m \sigma},
$$

where $r_{i m \sigma}=\left(z_{i m \sigma}\right)^{2}=r_{A m \sigma}$ and $r_{B m \sigma}$ for $i \in A$ and $i \in B$, respectively. After a simple calculation, we get $\Phi_{2}$ given by

$$
\Phi_{2}=\int d \varepsilon f(\varepsilon)(1 / \pi) \operatorname{Im} \sum_{m k \sigma} \ln \left(q_{m \sigma}^{2}\left[X_{A m \sigma}(\varepsilon) X_{B m \sigma}(\varepsilon)-\varepsilon_{k}^{2}\right]\right)
$$

where the band-narrowing factor, $q_{m \sigma}$, is given by

$$
q_{m \sigma}=z_{A m \sigma} z_{B m \sigma}=z_{m \sigma} z_{m-\sigma}=\sqrt{r_{m \sigma} r_{m-\sigma}},
$$

because $z_{A m \sigma}=z_{B m-\sigma}=z_{m \sigma}$ and $r_{A m \sigma}=r_{B m-\sigma}=r_{m \sigma}$. 
The mean-field free energy is obtained from the saddle-point values of the integration variables for which the variational conditions yield the following simultaneous equations:

$$
\begin{gathered}
n_{i m}=\sum_{\sigma} n_{i m \sigma} \\
m_{i m}=\sum_{\sigma} \sigma n_{i m \sigma} \\
\mu-\nu_{i m}+\sum_{\sigma} R_{i m \sigma}\left(\partial r_{i m \sigma} / \partial n_{i m}\right)=0, \\
\xi_{i m}+\sum_{\sigma} R_{i m \sigma}\left(\partial r_{i m \sigma} / \partial m_{i m}\right)=0, \\
2 U_{0}+\sum_{m \sigma} R_{i m \sigma}\left(\partial r_{i m \sigma} / \partial d_{i 0}\right)=0, \\
2 U_{1}+\sum_{m \sigma} R_{i m \sigma}\left(\partial r_{i m \sigma} / \partial d_{i 1}\right)=0, \\
U_{2}+\sum_{m \sigma^{\prime}} R_{i m \sigma^{\prime}}\left(\partial r_{i m \sigma^{\prime}} / \partial d_{i \sigma}\right)=0, \\
2\left(U_{0}+U_{1}+U_{2}\right)+\sum_{m \sigma^{\prime}} R_{i m \sigma^{\prime}}\left(\partial r_{i m \sigma^{\prime}} / \partial t_{i \sigma}\right)=0 \\
2\left(U_{0}+U_{1}+U_{2}\right)+\sum_{m \sigma} R_{i m \sigma}\left(\partial r_{i m \sigma} / \partial f_{i}\right)=0
\end{gathered}
$$

In Eqs.(28)-(36), $R_{i m \sigma}$ and $n_{i m \sigma}$ are given by

$$
\begin{gathered}
R_{i m \sigma}=\partial \Phi_{2} / \partial r_{i m \sigma}=\int d \varepsilon f(\varepsilon)(-1 / \pi) \operatorname{Im}\left[\left(\Omega_{m \sigma} / r_{i m \sigma}\right) F_{0}\left(\Omega_{m \sigma}\right)\right] \\
n_{i m \sigma}=\int d \varepsilon f(\varepsilon) \rho_{i m \sigma}(\varepsilon)
\end{gathered}
$$

where the local densities of states at the site belonging to A and B sublattices are expressed by

$$
\begin{aligned}
\rho_{i m \sigma}(\varepsilon) & =(-1 / \pi) \operatorname{Im}\left[K_{A m \sigma}(\varepsilon) / r_{A m \sigma}\right] \quad(i \in A), \\
& =(-1 / \pi) \operatorname{Im}\left[K_{B m \sigma}(\varepsilon) / r_{B m \sigma}\right] \quad(i \in B),
\end{aligned}
$$


with

$$
\begin{gathered}
K_{A n \sigma}(\varepsilon)=\left[X_{B m \sigma}(\varepsilon) / X_{A m \sigma}(\varepsilon)\right]^{1 / 2} F_{0}\left(\Omega_{m \sigma}\right), \\
K_{B n \sigma}(\varepsilon)=\left[X_{A m \sigma}(\varepsilon) / X_{B m \sigma}(\varepsilon)\right]^{1 / 2} F_{0}\left(\Omega_{m \sigma}\right), \\
\Omega_{m \sigma}(\varepsilon)=\left[X_{A m \sigma}(\varepsilon) X_{B m \sigma}(\varepsilon)\right]^{1 / 2} \\
F_{0}(\varepsilon)=\int d \varepsilon^{\prime} \rho_{0}\left(\varepsilon^{\prime}\right) /\left(\varepsilon-\varepsilon^{\prime}\right)
\end{gathered}
$$

$\rho^{0}(\varepsilon)$ being the unperturbed density of states. ${ }^{24}$

\section{NUMERICAL CALCULATIONS}

Numerical calculations have been performed for the simple-cubic model with nearestneighbor hoppings $t$. Input parameters for our calculations are the non-interacting density of states, $\rho_{0}(\varepsilon)$, the Coulomb and exchange interactions, $U$ and $J$, and the number of electrons per sub-band, $n$, which is unity for the half-filled case. We employed the approximate, analytic expression for $\rho_{0}(\varepsilon)$ of the simple-cubic lattice, given by ${ }^{25}$

$$
\begin{aligned}
\rho_{0}(\varepsilon) & =A\left[9-\omega^{2}\right]^{1 / 2}-C\left[1-\omega^{2}\right]^{1 / 2} \quad \text { for }|\omega| \leq 1, \\
& =A\left[9-\omega^{2}\right]^{1 / 2}-B\left[1-(|\omega|-2)^{2}\right]^{1 / 2}, \quad \text { for } 1<|\omega| \leq 3, \\
& =0 \quad \text { for }|\omega|>3,
\end{aligned}
$$

where $\omega=\varepsilon / 2 t, A / 2 t=0.101081, B / 2 t=0.128067$ and $C / 2 t=0.02$. The energy and the interactions are hereafter measured in units of a half of the total band width, $W / 2=6 t=1$. The ground-state energy without interactions calculated by using Eq.(44) is $\varepsilon_{0}=-0.3349$, which is in good agreement with the exact value of $-0.3341 .{ }^{16}$ Since the 
relations: $e=f$ and $p_{\sigma}=t_{\sigma}$ hold for the half-filled case, we have to self-consistently solve Eqs.(28)-(43) for nine quantities: $m, \xi, d_{0}, d_{1}, d_{\uparrow}, d_{\downarrow}, t_{\uparrow}, t_{\downarrow}$ and $f$, by using the NewtonRapson method. We performed the integrations given by Eqs.(37) and (38) with the use of the contour integral along the complex energy axis ${ }^{26}$ in order to reduce the computational time.

\section{A. $J=0$ Case}

We firstly show the calculated results for the vanishing exchange interaction $(J=$ 0 ), for which $d_{0}$ and $d_{1}$ are equivalent. Figure 1 shows the sublattice magnetization, $m$, as a function of $U$. The antiferromagnetic state is realized for an infinitesimally small interaction. The magnetic moment increases with increasing $U$ and asymptotically approaches the saturated value of $1.0 \mu_{B}$ as $U \rightarrow \infty$. Because of large fluctuations, $m$ in GA is much reduced by more than $50 \%$ than that in the Hartree-Fock approximation (HFA) at $U<1$.

Figure 2 shows the spin-dependent local densities of states for $U=1.0$. They have traces of the van Hove singularity of the simple-cubic density of states and clear energy gaps characteristic of the antiferromagnetic insulator. The energy gap in the GA $\left(\Delta_{\mathrm{GA}}=0.114\right)$ is much reduced compared with that in the HFA $\left(\Delta_{\mathrm{HFA}}=0.764\right)$. The $U$ dependence of the energy gap is plotted in Fig.1. Both $\Delta_{\mathrm{GA}}$ and $\Delta_{\mathrm{HFA}}$ increase with increasing $U$ because $\Delta=2 \xi, \xi$ being the staggered exchange field. The ratio defined by $a \equiv \Delta_{\mathrm{GA}} / \Delta_{\mathrm{HFA}}=\xi_{\mathrm{GA}} / \xi_{\mathrm{HFA}}$ is unity in the limits of $U \rightarrow 0$ and $U \rightarrow \infty$, and it has a broad minimum of $a=0.12$ at $U \sim 0.6$, above which $a$ again increases: $a=0.15,0.29$ and 0.54 for $U=1.0,1.5$ and 2.0, respectively. 
The band-narrowing factor, which becomes $q_{m \uparrow}=q_{m \downarrow}=q$ in our half-filled model, is shown as a function of $U$ in Fig.3. In the $\mathrm{P}$ state, $q$ monotonously decreases with increasing $U$ as shown by the dotted curve, and it vanishes at $U=U_{c}=12 \varepsilon_{0}=4.019$ where $U_{c}$ denotes the critical interaction for the MI transition., ${ }^{4,6,7}$ On the contrary, the $U$ dependence of $q$ in the $\mathrm{AF}$ state is quite different from that in the $\mathrm{P}$ state. When $U$ is increased from the zero value, $q$ of the AF state gradually departs from that of the P state, and it has the minimum value of 0.837 at $U=1.4$, above which $q$ increases again. The effect of electron correlation on the band-narrowing factor is not considerable although its effect on the energy gap (or the exchange field) is significant.

The $U$-dependence of the occupancies is shown in Fig.4. At $U=0$ all the occupancies are $0.0625\left(=1 / 2^{4}\right)$. When $U$ value is increased, only $d_{\uparrow}$ considerably increases, approaching unity for $U=\infty$ : $d_{0}\left(=d_{1}\right)$ and $t_{\uparrow}$ have small peaks at $U \sim 1$ but decrease for larger $U$.

The $U$ dependence of the ground-state energies, $E$, is shown in Fig.5. The groundstate energy of the $\mathrm{AF}$ state $\left(E_{\mathrm{AF}}\right)$ calculated by the $\mathrm{GA}$ is not only lower than that of the $\mathrm{P}$ state $\left(E_{\mathrm{P}}\right)$ obtained by the GA but also lower than that of the AF state calculated by the HFA. The difference: $\Delta E=E_{\mathrm{AF}}(\mathrm{GA})-E_{\mathrm{AF}}(\mathrm{HFA})$ expresses the energy gain by including the effect of fluctuations, and its maximum value is -0.056 at $U=0.95$. The HFA for the Néel state is a good description of the half-filled DHM in the limit of $U=\infty$.

\section{B. Finite $J$ Case}

Next we introduce the exchange interaction, $J$, into our calculation. Figure 6 shows the 
sublattice magnetization as a function of $U+J$ for various choices of the ratio: $J / U=0.1$, 0.2 and 0.3 . Note that the magnetization in the HFA is universal when it is plotted against $U+J$ because its exchange field is given by $\xi_{\mathrm{HFA}}=(1 / 2)(U+J) m_{\mathrm{HFA}}$. As the value of $J / U$ is increased, the sublattice magnetization is increased as expected. This fact is more clearly seen in Fig.7, where the sublattice magnetization and the band-narrowing factor for $U=1.0$ are plotted as a function of $J$. The sublattice magnetization of the GA, particularly near $J=0$, is much increased when the $J$ value is increased, although such an increase in $m$ is realized also in the HFA result, but very small.

The interaction dependence of the band narrowing-factor is shown in Fig.8. It was recently pointed out ${ }^{6,7}$ that, when $J$ is finite, the first-order MI transition is realized in the $\mathrm{P}$ state, as is shown by dotted curves; it occurs at $U+J=2.21,1.95$ and 1.83 for $J / U=0.1,0.2$ and 0.3 , respectively. Our calculation shows that the situation is quite different in AF state: $q$ decreases only slightly and never vanishes. The minimum values of $q$ are $0.939,0.965$ and 0.975 for $J / U=0.1,0.2$ and 0.3 , respectively.

Figure 9 shows the occupancies as a function of $U+J$ in the typical case of $J / U=0.1$. For $J>0$ the degeneracy between $d_{0}$ and $d_{1}$ is removed and we get $d_{0}<d_{1}$. When the interaction is increased, only $d_{\uparrow}$ has an appreciable value at $U+J>0.5$, as in the case shown in Fig.4.

Figure 10 shows the ground-state energies, $E_{\mathrm{AF}}(\mathrm{GA}), E_{\mathrm{AF}}(\mathrm{HFA})$ and $E_{\mathrm{P}}(\mathrm{GA})$, as a function of the interaction. We realized that $E_{\mathrm{AF}}(\mathrm{GA})$ is the lowest among the three for any $U$ investigated. The maximum difference of $\Delta E$ is -0.029 at $U=0.7$.

\section{CONCLUSION AND DISCUSSION}


To summarize, we have studied the antiferromagnetic ground state in the DHM, employing our slave-boson mean-field theory. ${ }^{7}$ Numerical calculations have shown that the MI transition does not take place in the antiferromagnetic solution for the half-filled DHM except at the vanishing interaction point, which arises form a peculiarity due to the perfect nesting in the model. This is in contrast with the result in its paramagnetic solution, ${ }^{6,7}$ but is the same as the half-filled $\mathrm{SHM},{ }^{21}$ as was discussed in the Introduction. Except at $U=J=0$, the stable state is the antiferromagnetic insulator, whose energy gap is much reduced by electron correlation.

It is worth to make a brief comparison between the results of the DHM and SHM. Dashed lines in Figs.1, 3, 6 and 8 show the interaction dependence of $m, \Delta$ and $q$ of the SHM. ${ }^{21}$ When we compare these results with the corresponding ones of the DHM, we notice that both the results are very similar provided the exchange interaction is not small; $J / U>0.2$. When $J$ is small, however, $m, \Delta$ and $q$ in the DHM are fairly smaller than those in the SHM. Figure 7 shows that the exchange interaction effectively works to increase the magnitude of sublattice moment in the DHM.

In order to more investigate the role of the exchange interaction on antiferromagnetism in the DHM, we have repeated a numerical calculation for the negative $J$, although $J$ is conventionally taken to be positive. We notice in Fig.7 that when the negative exchange interaction is included, the sublattice magnetization for $U=1.0$ is considerably reduced and it disappears for $J / U<-0.05$. Figure 11 shows the sublattice magnetization and the band narrowing factor as a function of $U+J$ for $J / U=-0.02$. When the interaction is increased, the magnetization first increases at $U+J<1.2$ but decreases at larger interaction. Surprisingly the antiferromagnetic state disappears at $U+J \geq 2.1$, which is 
in strong contrast with the HFA result shown by the dotted curve. Figure 12 shows the interaction dependence of the occupancies. The behavior of the double occupancies in the negative $J$ case is rather different from that in the positive $J$ case shown in Fig.9. When $J$ is negative, the doubly occupied state with the opposite spin between the different subbands is less favorable than that within the same subband. Furthermore the triplet state expressed by $d_{\uparrow}$ (or $d_{\downarrow}$ ) becomes less stable than the singlet state expressed by $d_{0}$ or $d_{1}$, which works to suppress the antiferromagnetism. We get $d_{0}>d_{1}>d_{\uparrow}\left(=d_{\downarrow}\right)$ in the paramagnetic state at $U+J>2.1$. The first-order MI transition occurs at $U+J=2.68$. A material with the anti-Hund-rule coupling $(J<0)$ would show the unusual behavior if it exists.

The model Hamiltonian adopted in our study (Eq.(1)) is relevant to systems with partially filled narrow degenerate bands. A typical example is $\mathrm{V}_{2} \mathrm{O}_{3}$, which is an antiferromagnetic insulator (AFI) in the ground state and which shows the MI transition between AFI, paramagnetic metal and paramagnetic insulator as a function of the temperature, the pressure and/or the chemical substitution. This phase diagram can be qualitatively understood with the SHM. ${ }^{21,27}$ The degenerate Hubbard model has much variety than the SHM because it has an additional, orbital degree of freedom. It may show the orbital ordering besides the spin ordering. The stability of the different phases in the degenerate band depends not only on the values of the various interactions included in the model Hamiltonian but also on the temperature. It would be interesting to investigate the temperature-interaction phase diagram of the DHM, by generalizing our approach ${ }^{21}$ in which the effects of electron correlation and thermal spin fluctuations are properly taken into account. 


\section{Acknowledgment}

This work is partly supported by a Grant-in-Aid for Scientific Research from the Japanese Ministry of Education, Science and Culture. 


\section{References}

[1] M. C. Gutzwiller, Phys. Rev. Lett. 10, 159 (1963); Phys. Rev. 137, A1726 (1965).

[2] J. Kanamori, Prog. Theor. Phys. 30, 235 (1963).

[3] J. Hubbard, Proc. Roy. Soc. London A281, 401 (1964).

[4] J. P. Lu, Phys. Rev. B 49, 5687 (1994).

[5] T. Okabe, J. Phys. Soc. Jpn. 65, 1056 (1996).

[6] J. Bünemann and W. Weber, Phys. Rev. B 55, R4011 (1997).

[7] H. Hasegawa, J. Phys. Soc. Jpn. 66, 1391 (1997); preprint (SISSA: condmat/9704168).

[8] R. Frésard and G. Kotliar, preprint (SISSA:. cond-mat/9612172).

[9] M. J. Rozenberg, preprint (SISSA:. cond-mat/960976).

[10] G. Kotliar and H. Kajueter, Phys. Rev. B 54, R14221 (1996).

[11] K. A. Chao and M. C. Gutzwiller, Phys. Rev. B 4, 4034 (1971).

[12] V. Dorin and P. Schlottmann, Phys. Rev. 47, 5095 (1993).

[13] G. Kotliar and A. E. Ruckenstein, Phys. Rev. Lett. 57, 1362 (1986).

[14] W. F. Brinkman and T. M. Rice, Phys. Rev. B 2, 4302 (1970).

[15] T. A. Kaplan, P. Horsch and P. Fulde, Phys. Rev. Lett. 49, 889 (1982). 
[16] H. Yokoyama and H. Shiba, J. Phys. Soc. Jpn. 56, 1490 (1987).

[17] W. Metzner and D. Vohlhardt, Phys. Rev. Letter 59, 121 (1987); Phys. Rev. B 37, $7382(1988)$.

[18] H. Yokoyama and H. Shiba, J. Phys. Soc. Jpn. 56, 3582 (1987).

[19] W. Metzner, Z. Phys. B 77, 253 (1989).

[20] F. Gebhard, Phys. Rev. B 41 (1990) 253.

[21] H. Hasegawa, J. Phys. Cond. Matter. 1, 9325 (1989); Prog. Theor. Phys. Suppl.101, 463 (1990).

[22] H. Hasegawa, Phys. Rev. 41, 9168 (1990).

[23] H. Shiba, Prog. Theor. Phys. 46, 77 (1971).

[24] The equations relevant to the ferromagnetic state are given if we set $X_{A m \sigma}=X_{B m \sigma}=$ $X_{m \sigma}, K_{A m \sigma}(\varepsilon)=K_{B m \sigma}(\varepsilon)=F_{0}\left(X_{m \sigma}\right)$ and $q_{m \sigma}=z_{m \sigma}^{2}$ in Eqs.(26)-(43).

[25] T. Tonegawa, Prog. Theor. Phys. 51, 1293 (1974).

[26] A. R. Williams, P. J. Feilbelman and N. D. Lang, Phys. Rev. B 30, 3113 (1984); R. Zeller, J. Deutz and P. H. Dederichs, Solid State Commun. 44, 993 (1984).

[27] M. Jarrell, Phys. Rev. Lett. 69, 168 (1992);

A. Georges and W. Krauth, Phys. Rev. B 48, 7167 (1993);

M. J. Rozenberg, G. Kotliar, and X. Y. Zhang, Phys. Rev. B 49, 10181 (1994). 


\section{Figure Captions}

Fig. 1 The sublattice magnetization, $m$, and the energy gap, $\Delta$, as a function of $U$ of the DHM with $J=0$ in the GA (solid curve) and in the HFA (dotted curve), the results of the SHM being shown by the dashed curve (Ref.21).

Fig. 2 The spin-dependent local densities of states for $U=1.0$ and $J=0.0$ : $\uparrow$-spin (solid curve) and $\downarrow$-spin (dashed curve) components in the GA, and $\uparrow$-spin (dot-dashed curve) and $\downarrow$-spin (dottedcurve) components in the HFA.

Fig. 3 The band-narrowing factor, $q$, as a function of $U$ of the DHM with $J=0$ in the AF state (solid curve) and in the $\mathrm{P}$ state (dotted curve), the result of the SHM in the AF state being shown by the dashed curve (Ref.21).

Fig. 4 The occupancies as a function of $U$ with $J / U=0$, the result of $d_{\uparrow}$ divided by a factor of ten being plotted by the dot-dashed curve.

Fig. 5 The $U$-dependence of the ground-state energies $E$ of the AF state with $J=0$ in the GA (solid curve) and in the HFA (dashed curve), and their difference: $\Delta E=$ $E_{\mathrm{AF}}(G A)-E_{\mathrm{AF}}(H F A)$. The result of the $\mathrm{P}$ state in the GA is shown by the dotted curve.

Fig. 6 The sublattice magnetization, $m$, as a function of $U+J$ of the DHM with various $J / U$ values in the GA (solid curve) and in the HFA (dotted curve), the result of the SHM being shown by the dashed curve (Ref.21). 
Fig. 7 The sublattice magnetization for $U=1.0$ as a function of $J$ in the GA (solid curve) and in the HF (dotted curve), the result of the band narrowing factor being also plotted (chain curve).

Fig. 8 The band-narrowing factor, $q$, as a function of $U+J$ of the DHM with various $J / U$ values in the AF state (solid curve) and in the $\mathrm{P}$ state (dotted curve), the result of the SHM in the AF state being shown by the dashed curve (Ref.21).

Fig. 9 The occupancies for $J / U=0.1$ as a function of $U+J$, the result of $d_{\uparrow}$ divided by a factor of ten being plotted by the chain curve.

Fig. 10 The interaction dependence of the ground-state energies of the AF state with $J / U=0.1$ in the GA (solid curve) and in the HF (dashed curve), and their difference: $\Delta E=E_{\mathrm{AF}}(G A)-E_{\mathrm{AF}}(H F A)$. The result of the $\mathrm{P}$ state in the GA is shown by the dotted curve.

Fig. 11 The sublattice magnetization, $m$, as a function of $U+J$ with $J / U=-0.02$ in the GA (solid curve) and in the HFA (dotted curve), the band narrowing factor, $q$, being shown by the chain curve.

Fig. 12 The occupancies for $J / U=-0.02$ as a function of $U+J$. 